Proyecciones Journal of Mathematics

Vol. 38, No 1, pp. 163-175, March 2019.

Universidad Católica del Norte

Antofagasta - Chile

\title{
Nonlinear maps preserving certain subspaces
}

\author{
H. Benbouziane \\ University Sidi Mohamed Ben Abdellah, Morocco \\ $Y$. Bouramdane \\ University Sidi Mohamed Ben Abdellah, Morocco \\ and \\ M. Ech-Chérif El Kettani \\ University Sidi Mohamed Ben Abdellah, Morocco \\ Received : April 2018. Accepted : May 2018
}

\begin{abstract}
Let $X$ be a Banach space and let $\mathcal{B}(X)$ be the Banach algebra of all bounded linear operators on $X$. We characterise surjective (not necessarily linear or additive) maps $\phi: \mathcal{B}(X) \rightarrow \mathcal{B}(X)$ such that $F(\phi(A) \diamond \phi(B))=F(A \diamond B)$ for all $A, B \in \mathcal{B}(X)$ where $F(A)$ denotes any of $R(A)$ or $N(A)$, and $A \diamond B$ denotes any binary operations $A-B$, $A B$ and $A B A$ for all $A, B \in \mathcal{B}(X)$.
\end{abstract}

Subjclass [2010]: Primary 47B49; Secondary 47B48,46H05.

keywords : Kernel operator, Nonlinear preservers problem, Range operator. 


\section{Introduction}

Throughout this note, let $X$ be an infinite dimensional Banach space over the field $\mathbf{K}$ ( where $\mathbf{K}$ is $\mathbf{R}$ or $\mathbf{C}$ ), and $\mathcal{B}(X)$ be the Banach algebra of all bounded linear operators on $X$. For $A \in \mathcal{B}(X)$ denote by $R(A)$ the range of $A, N(A)$ its kernel and $A^{*}$ its adjoint acting on the dual space $X^{*}$ of $X$. The hyper-kernel and the hyper-range of $A \in \mathcal{B}(X)$ are defined respectively by

$$
\mathcal{N}^{\infty}(A):=\bigcup_{n \in \mathbf{N}} N\left(A^{n}\right) \text { and } \mathcal{R}^{\infty}(A):=\bigcap_{n \in \mathbf{N}} R\left(A^{n}\right)
$$

For $x \in X$ and $f \in X^{*}$, the operator of rank at most one is denoted, as usual, by $(x \otimes f)(y):=f(y) x$ for all $y \in X$. The set of all rank one operators is denoted by $\mathcal{F}_{1}(X)$. Preserver problems aim to characterize linear or nonlinear maps on operator algebras preserving certain properties, subsets, or relations. It has been initiated in [7], and continuous by several authors, see for example[1, 2, 3, 5, 6, 8], and the references therein. In [8], the forms of surjective additive maps $\phi: \mathcal{B}(X) \rightarrow \mathcal{B}(X)$ preserving the range's codimension or the kernel's dimension are determined. In particular the author shows that a surjective additive map $\phi: \mathcal{B}(X) \rightarrow \mathcal{B}(X)$ preserves the range (respectively the kernel) if and only if there is an invertible operator $T \in \mathcal{B}(X)$ such that $\phi(A)=A T$ (respectively $\phi(A)=T A$ ) for all $A \in$ $\mathcal{B}(X)$. Also, in [6] surjective additive maps preserving the hyper range or the hyper kernel are determined. It is shown that a surjective additive map $\phi: \mathcal{B}(X) \rightarrow \mathcal{B}(X)$ satisfies $\mathcal{N}^{\infty}(\phi(A))=\mathcal{N}^{\infty}(A)\left(\right.$ or $\mathcal{R}^{\infty}(\phi(A))=\mathcal{R}^{\infty}(A)$ if and only if there exists a nonzero constant $c$ such that $\phi(A)=c A$ for all $A \in \mathcal{B}(X)$. Here we propose to treat the nonlinear case of these results. We will replace the linearity assumption and the preserving property of the map $\phi$ by the weaker condition $R(\phi(A)-\phi(B))=R(A-B)($ or $N(\phi(A)-\phi(B))=$ $N(A-B)$ ). Moreover, we examine more related problems by replacing the difference in assumption by product or triple product.

\section{Preliminaries}

In this section, we collect some lemmas that will be used in the proof of our main results. We begin by giving the range and kernel of rank one operators.

Lemma 2.1. Let $x \in X$ be a nonzero vector and $f \in X^{*}$ be a nonzero linear form. We have

$$
\text { 1. } R(x \otimes f)=\operatorname{span}\{x\} \text { and } N(x \otimes f)=N(f) \text {. }
$$


2. If $f(x)=1$, then $N(I-x \otimes f)=R(x \otimes f)=\operatorname{span}\{x\}$ and $R(I-x \otimes$ $f)=N(x \otimes f)=N(f)$.

3. If $f(x) \neq 0$ then $\mathcal{R}^{\infty}(x \otimes f)=R(x \otimes f)=\operatorname{span}\{x\}$.

Proof. [6, Lemma 2.1]

Next result, quoted from [2], characterizes surjective maps preserving the surjectivity or injectivity of the difference of operators, (i,e) the maps $\phi$ such that $A-B$ injective (resp. surjective) if and only $\phi(A)-\phi(B)$ injective (resp. surjective).

Theorem 2.2. Let $X$ be a complex Banach space, and let $\phi: B(X) \rightarrow$ $B(X)$ be a surjective map. If $\phi$ preserves injectivity (resp. surjectivity) of the difference of operators in both directions. Then one of the following statements holds.

1. There are a bounded invertible operators $T, S: X \rightarrow X$ both linear, or both conjugate linear such that $\phi(A)=T A S+\phi(0)$ for all $A \in B(X)$.

2. There are a bounded invertible operators $T: X^{*} \rightarrow X$ and $S: X \rightarrow$ $X^{*}$ both linear, or both conjugate linear such that $\phi(A)=T A^{*} S+$ $\phi(0)$ for all $A \in B(X)$.

The last case may occur only if $X$ is reflexive.

The following lemma determines the structure of surjective maps preserving the zero product.

Lemma 2.3. Let $X$ be a real or complex Banach space, and let $\phi: B(X) \rightarrow$ $B(X)$ be a surjective map that satisfies

(2.1) $\phi(A) \phi(B)=0$ if and only if $A B=0$ for all $A, B \in B(X)$.

Then there is a scalar function $\varphi: B(X) \rightarrow \mathbf{K} \backslash\{0\}$ and a bounded invertible linear, or conjugate linear, operator $T: X \rightarrow X$ such that $\phi(A)=$ $\varphi(A) T A T^{-1}$ for all $A \in \mathcal{F}_{1}(X)$. 
Proof. [4, Lemma 2.2]

We close this section with the following result which determines the structure of surjective maps preserving pairs having zero triple product.

Lemma 2.4. [5] Let $X$ be a real or complex Banach space, and let $\phi$ : $B(X) \rightarrow B(X)$ be a surjective map that satisfies

$\phi(A) \phi(B) \phi(A)=0$ if and only if $A B A=0$ for all $A, B \in B(X)$.

Then there is a scalar functional $\varphi: B(X) \rightarrow \mathbf{K} \backslash\{0\}$ such that one of the following statements holds.

1. There is a bounded invertible linear, or a conjugate linear, operator $T: X \rightarrow X$ such that $\phi(A)=\varphi(A) T A T^{-1}$ for all $A \in B(X)$.

2. There is a bounded invertible linear, or a conjugate linear, operator $T: X^{*} \rightarrow X$ such that $\phi(A)=\varphi(A) T A^{*} T^{-1}$ for all $A \in B(X)$.

\section{Nonlinear maps preserving the kernel}

We begin this section by stating and proving the following lemma which will be used in the sequel.

Lemma 3.1. Let $A, B \in B(X)$. If $N(R A)=N(R B)$ for all rank one operators $R$, then there is a nonzero scalar $c$ such that $A=c B$.

Proof. Let $A, B \in B(X)$. Choose $x \in X$ a nonzero vector and $f \in X^{*}$ nonzero linear form. If $N(R A)=N(R B)$ for all rank one operators $R$. It follows, for $R=x \otimes f$, that $N(x \otimes f A)=N(x \otimes f B)$, which is equivalent to $N\left(x \otimes A^{*} f\right)=N\left(x \otimes B^{*} f\right)$. Thus $N\left(A^{*} f\right)=N\left(B^{*} f\right)$. So, $A^{*} f$ and $B^{*} f$ are linearly dependent for every nonzero linear form $f \in X^{*}$. By hypothesis one see that $N\left(A^{*}\right)=N\left(B^{*}\right)$. Consequently $A^{*}$ and $B^{*}$ are linearly dependent. Thus $A$ and $B$ are linearly dependent; as desired.

Now, we are able to state and prove our main results, we start by the following theorem characterizing the surjective maps that preserve the kernel of product of operators.

Theorem 3.2. Let $X$ be a real or complex Banach space. A surjective $\operatorname{map} \phi: B(X) \rightarrow B(X)$ satisfies

$$
N(\phi(A) \phi(B))=N(A B) \text { for all } A, B \in B(X),
$$


if and only if there exists $\varphi: B(X) \rightarrow \mathbf{K} \backslash\{0\}$ such that $\phi(A)=\varphi(A) A$ for all $A \in B(X)$.

Proof. The 'if' part is easily verified. To prove the 'only if' part, assume that $\phi$ satisfies the equation (3.1). In particular $N(\phi(A) \phi(B))=X$ if and only if $N(A B)=X$ for all $A, B \in B(X)$, so $\phi$ satisfies the equation (2.1). It follows, by Lemma 2.3, that there is a scalar function $\varphi: B(X) \rightarrow \mathbf{K} \backslash\{0\}$ and there is a bounded invertible linear, or conjugate linear, operator $T$ : $X \rightarrow X$ such that $\phi(A)=\varphi(A) T A T^{-1}$ for all $A \in \mathcal{F}_{1}(X)$.

In the sequel we will prove that $T A$ and $\phi(A)$ are linearly dependent for all $A \in B(X)$ and $T=c I$ for some nonzero scalar $c$. Fix $A \in B(X)$, and let $R$ be a rank one operator. We have

$$
N(R A)=N(\phi(R) \phi(A))=N\left(\varphi(R) T R T^{-1} \phi(A)\right)=N\left(R T^{-1} \phi(A)\right) .
$$

Thus, by Lemma 3.1, $A$ and $T^{-1} \phi(A)$ are linearly dependent. Then there exists a nonzero scalar $c \in \mathbf{K}$ such that $\phi(A)=c T A$ for all $A \in B(X)$. Note that $c$ depends on $A$ and $T$ and from the equation (3.1) $c$ cannot be 0 for $A \neq 0$. It remains to prove that $T=\alpha I$ for some nonzero scalar $\alpha$. Indeed, set $A=I-x \otimes f$ where $x \in X$ and $f \in X^{*}$ such that $f(x)=1$ and $B=I$, since $N(A B)=N(\phi(A) \phi(B))=N(A T B)$ for all $A, B \in B(X)$, it follows that

$$
\begin{aligned}
\operatorname{span}\{x\} & =N(I-x \otimes f) \\
& =N((I-x \otimes f) T) \\
& =N(T-x \otimes f T) \\
& =N\left(T\left(I-T^{-1} x \otimes f T\right)\right) \\
& =N\left(I-T^{-1} x \otimes f T\right) \\
& =\operatorname{span}\left\{T^{-1} x\right\} .
\end{aligned}
$$

This implies that $T^{-1} x$ and $x$ are linearly dependent. So $T=\alpha I$ for some nonzero scalar $\alpha$. We conclude that $\phi(A)=\varphi(A) A$ for all $A \in B(X)$.

We end this section with the following result that characterizes surjective maps preserving the kernel of the difference of operators.

Theorem 3.3. Let $X$ be a complex Banach space. A surjective map $\phi$ : $B(X) \rightarrow B(X)$ satisfies 


$$
N(\phi(A)-\phi(B))=N(A-B) \text { for all } A, B \in B(X),
$$

if and only if there exists $T \in B(X)$ invertible such that $\phi(A)=T A+\phi(0)$ for all $A \in B(X)$.

Proof. The necessarily condition is easily verified since $T$ is injective. Conversely, assume that $\phi$ satisfies the equation (3.2). Then $\phi(A)-\phi(B)$ is injective if and only if $A-B$ is injective for all $A, B \in B(X)$. It follows that $\phi$ takes one of the forms given by Theorem 2.2 .

Assume that $\phi$ takes the first one that is $\phi(A)=T A S+\phi(0)$ for all $A \in B(X)$, where $T, S: X \rightarrow X$ are bounded invertible both linear, or both conjugate linear. Let us prove that $S=c I$ for some nonzero scalar $c$. To do that, consider the surjective map

$$
\psi(A)=\phi(A)-\phi(0),(A \in B(X)),
$$

which satisfies the equation (3.2) with $\psi(0)=0$.

Note that $\psi(A)=T A S$ and $N(\psi(A))=N(A)$ for all $A \in B(X)$. For a rank one operator $R \in B(X)$ we have

$$
N(R)=N(\psi(R))=N(T R S)=N(R S) .
$$

Thus, by Lemma 3.1, $S=c I$ for some nonzero scalar $c$. Therefore $\phi(A)=$ $T A+\phi(0)$.

To finish the proof it remains to show that the second form can not occur. Indeed, assume that

$$
\phi(A)=T A^{*} S+\phi(0),(A \in B(X)),
$$

where $T: X^{*} \rightarrow X$ and $S: X \rightarrow X^{*}$ are bounded invertible both linear, or both conjugate linear. Since $X$ is reflexive, we can find an injective and non-surjective operator $A \in B(X)$, see $[3,9]$. Then, there is an operator $U \in B(X)$ such that $A=\phi(U)$ with $U$ is injective, because $\phi$ is surjective. For a nonzero vector $y \in X$ let $f \in X^{*}$ with $f=T^{-1} y$ and $x \in X \backslash\{0\}$ such that $f(x)=1$. We have

$$
\begin{aligned}
N\left(A-T(U x \otimes f)^{*} S\right) & =N(\phi(U)-\phi(U x \otimes f)) \\
& =N(U-U x \otimes f) \\
& =N(I-x \otimes f) \\
& =\operatorname{span}\{x\} .
\end{aligned}
$$


Consequently, $A-T(U x \otimes f)^{*} S$ is not injective. Since $A$ is injective we deduce that

$$
\operatorname{span}\{T f\}=R\left(T(U x \otimes f)^{*} S\right) \subset R(A) .
$$

Thus $y=T f \in R(A)$ and so $A$ is surjective which is a contradiction. This completes the proof.

\section{Nonlinear maps preserving the range}

The first theorem of this section characterizes the surjective maps preserving the range of the difference of operators.

Theorem 4.1. Let $X$ be a complex Banach space. A surjective map $\phi$ : $B(X) \rightarrow B(X)$ satisfies

$$
R(\phi(A)-\phi(B))=R(A-B) \text { for all } A, B \in B(X),
$$

if and only if there exists $S \in B(X)$ invertible such that $\phi(A)=A S+\phi(0)$ for all $A \in B(X)$.

Proof. The necessarily condition is easily verified since the operators $S$ is surjective. Conversely, assume that $R(\phi(A)-\phi(B))=R(A-$ $B)$ for all $A, B \in B(X)$, then $R(\phi(A)-\phi(B))=X$ if and only if $R(A-B)=X$ for all $A, B \in B(X)$. Thus $\phi(A)-\phi(B)$ is surjective if and only if $A-B$ is surjective for all $A, B \in B(X)$, it follows, by Theorem 2.2 , that one of the following assertions holds.

1. There are two bounded invertible both linear, or both conjugate linear, operators $T, S: X \rightarrow X$ such that $\phi(A)=T A S+\phi(0)$ for all $A \in B(X)$

2. There are two bounded invertible both linear, or both conjugate linear, operators $T: X^{*} \rightarrow X$ and $S: X \rightarrow X^{*}$ such that $\phi(A)=$ $T A^{*} S+\phi(0)$ for all $A \in B(X)$.

Let us prove that $\phi$ can not takes the second form. Assume for sake of contradiction that $\phi$ takes a such form. Note that the map

$$
\psi(A):=\phi(A)-\phi(0)
$$

satisfies the equation (4.1) with $\psi(0)=0$. For $x \in X$ and $f \in X^{*}$, we have 


$$
\begin{aligned}
\operatorname{span}\{x\} & =R(x \otimes f) \\
& =R(\psi(x \otimes f)) \\
& \left.=R\left(T(x \otimes f)^{*} S\right)\right) \\
& =R(T f \otimes J x S) \quad\left(J \text { is the natural embedding of } X \text { to } X^{* *}\right) \\
& =\operatorname{span}\{T f\} .
\end{aligned}
$$

Hence, $x$ and $T f$ are linearly dependent for every $x \in X$ and $f \in$ $X^{*}$. Which contradicts the fact that $T$ is bijective and $X$ with dimension greatest than 2 . Thus $\phi$ takes the first form.

It remains to prove that $T=c I$ for some nonzero scalar $c$. To do that, choose $x \in X$ and $f \in X^{*}$. We have

$$
\begin{aligned}
\operatorname{span}\{x\} & =R(x \otimes f) \\
& =R(\psi(x \otimes f)) \\
& =R(T x \otimes f S) \\
& =\operatorname{span}\{T x\} .
\end{aligned}
$$

Which implies that for all $x \in X, x$ and $T x$ are linearly dependent. Thus, there is a nonzero scalar $c$ such that $T=c I$.

Finally, dividing $S$ by $c$ if necessary, we see that $\phi(A)=A S+\phi(0)$ and $S \in B(X)$. This finishes the proof.

With no extra efforts, the same proof as the one of the above theorem yields the same conclusion by using the hyper-range of operator in the equation (4.1) instead of the range of operator.

Theorem 4.2. Let $X$ be a complex Banach space. A surjective map $\phi$ : $B(X) \rightarrow B(X)$ satisfies

$$
\mathcal{R}^{\infty}(\phi(A)-\phi(B))=\mathcal{R}^{\infty}(A-B) \text { for all } A, B \in B(X),
$$

if and only if there exist $S \in B(X)$ invertible such that $\phi(A)=A S+\phi(0)$ for all $A \in B(X)$.

The following result characterises the surjective maps preserving the range of operator product. 
Theorem 4.3. Let $X$ be a real or complex Banach space. A surjective map $\phi: B(X) \rightarrow B(X)$ satisfies

$$
R(\phi(A) \phi(B))=R(A B) \text { for all } A, B \in B(X),
$$

if and only if there exists $\varphi: B(X) \rightarrow \mathbf{K} \backslash\{0\}$ such that $\phi(A)=\varphi(A) A$ for all $A \in B(X)$.

Proof. The necessarily condition is easily verified. Conversely, assume that $R(\phi(A) \phi(B))=R(A B)$ for all $A, B \in B(X)$. Then $R(\phi(A) \phi(B))=$ $\{0\}$ if and only if $R(A B)=\{0\}$ for all $A, B \in B(X)$. It follows that $\phi(A) \phi(B)=0$ if and only if $A B=0$ for all $A, B \in B(X)$. Thus by Lemma 2.3, there exist a scalar function $\varphi: B(X) \rightarrow \mathbf{K} \backslash\{0\}$ and a bounded invertible linear, or conjugate linear, $T: X \rightarrow X$ such that $\phi(A)=$ $\varphi(A) T A T^{-1}$ for all $A \in \mathcal{F}_{1}(X)$. Let us show that $T=c I$ for some nonzero scalar $c$. To do that consider $x \in X$ a nonzero vector, choose $f \in X^{*}$ such that $f(x)=1$, we have

$$
\begin{aligned}
\operatorname{span}\{x\} & =R(x \otimes f) \\
& =R(x \otimes f x \otimes f) \\
& =R(\phi(x \otimes f) \phi(x \otimes f)) \\
& =R\left(\varphi(x \otimes f)^{2} T x \otimes f T^{-1}\right) \\
& =\operatorname{span}\{T x\} .
\end{aligned}
$$

It follows that $x$ and $T x$ ar linearly dependent for all $x \in X$. Then there exists a nonzero scalar $c$ such that $T=c I$. Thus

$$
\phi(A)=\varphi(A) A \text { for all } A \in \mathcal{F}_{1}(X) .
$$

To finish the proof, let $A$ be an arbitrary operator in $B(X)$, and let us show that $A$ and $\phi(A)$ are linearly dependent. For every $x \in X$ and $f \in X^{*}$ we have

$$
\begin{aligned}
\operatorname{span}\{A x\} & =R(A x \otimes f) \\
& =R(\phi(A) \phi(x \otimes f)) \\
& =R(\varphi(x \otimes f) \phi(A) x \otimes f) \\
& =\operatorname{span}\{\phi(A) x\} .
\end{aligned}
$$


Thus $A$ and $\phi(A)$ are linearly dependent, and the proof is complete.

We close this paper with a theorem which characterizes maps preserving the range of the triple product of operators.

Theorem 4.4. Let $X$ be a real or complex Banach space. A surjective map $\phi: B(X) \rightarrow B(X)$ satisfies

$$
R(\phi(A) \phi(B) \phi(A))=R(A B A) \text { for all } A, B \in B(X),
$$

if and only if there exists $\varphi: B(X) \rightarrow \mathbf{K} \backslash\{0\}$ such that $\phi(A)=\varphi(A) A$ for all $A \in B(X)$.

Proof. The necessarily condition is easily verified. Conversely, assume that $\phi$ satisfies the equation (4.4). In particular $R(\phi(A) \phi(B) \phi(A))=\{0\}$ if and only if $R(A B A)=\{0\}$ for all $A, B \in B(X)$, it then follows that $\phi(A) \phi(B) \phi(A)=0$ if and only if $A B A=0$ for all $A, B \in B(X)$. Thus, by Lemma 2.4, there is a scalar function $\varphi: B(X) \rightarrow \mathbf{K} \backslash\{0\}$ such that one of the following assertions holds.

1. There is a bounded invertible linear, or conjugate linear, $T: X \rightarrow X$ such that $\phi(A)=\varphi(A) T A T^{-1}$ for all $A \in B(X)$.

2. There is a bounded invertible linear, or conjugate linear, $T: X^{*} \rightarrow X$ such that $\phi(A)=\varphi(A) T A^{*} T^{-1}$ for all $A \in B(X)$.

The second case can not occur. Indeed, if $\phi(A)=\varphi(A) T A^{*} T^{-1}$, then $\phi(I)=\varphi(I) I$. We can write

$$
R(A)=R(\phi(A)) \text { for all } A \in B(X) .
$$

In particular for all $x \in X$ and $f \in X^{*}$ we have

$$
\begin{aligned}
\operatorname{span}\{x\} & =R(x \otimes f) \\
& =R(\phi(x \otimes f)) \\
& =R\left(\varphi(x \otimes f) T(x \otimes f)^{*} T^{-1}\right) \\
& =R\left(T f \otimes J x T^{-1}\right) \\
& =\operatorname{span}\{T f\} .
\end{aligned}
$$

Thus, $x$ and $T f$ are linearly dependent for every $x \in X$ and $f \in X^{*}$, this contradict the fact that $T$ is bijective and $X$ with dimension greatest than 2. Therefore $\phi$ takes the first form. 
It remains to prove that $T=c I$. As the above consideration, we have

$$
R(A)=R(\phi(A)) \text { for all } A \in B(X) .
$$

In particular for $x \in X$ and $f \in X^{*}$ we have

$$
\begin{aligned}
\operatorname{span}\{x\} & =R(x \otimes f) \\
& =R(\phi(x \otimes f)) \\
& =R\left(\varphi(I)^{2} T x \otimes f T^{-1}\right) \\
& =\operatorname{span}\{T x\} .
\end{aligned}
$$

It follows that for all $x \in X, x$ and $T x$ are linearly dependent. Thus, there is a nonzero scalar $c$ such that $T=c I$.

We conclude that there exists $\varphi: B(X) \rightarrow \mathbf{K} \backslash\{0\}$ such that $\phi(A)=$ $\varphi(A) A$ for all $A \in B(X)$; as desired.

Acknowledgements. The authors would to thank warmly the anonymous referee for carefully reading the paper and for valuable suggestions.

\section{References}

[1] H. Benbouziane, Y. Bouramdane, M. Ech-Cherif El Kettani, A. Lahssaini: Nonlinear commutant preservers, Linear and Multilinear Algebra 463 No.3, pp. 593-601, (2018).

[2] A. Bourhim, J. Mashreghi and A. Stepanyan, Nonlinear maps preserving the minimum and surjectivity moduli, Linear Algebra Appl. 463, pp. 171-189, (2014).

[3] J. Cui, J. Hou, Additive maps on standard operator algebras preserving invertibilities or zero divisors, Linear Algebra Appl. 359, pp. 219-233, (2003).

[4] J. Cui, J. Hou, Maps leaving functional values of operator products invariant, Linear Algebra Appl. 428, pp. 1649-1663, (2008). 
[5] G. Doboviek, B. Kuzma, G. Lenjak, C. K. Li, T. Petek, Mappings that preserve pairs of operators with zero triple Jordan product, Linear Algebra Appl. 426, pp. 255-279, (2007).

[6] M. Elhodaibi and A. Jaatit, On Additive maps preserving the hyperrange or hper-kernel of operators, Int. Math. Forum 7 No. 25-28, pp. 1223-1231, (2012).

[7] G. Frobenius, Über die Darstellung der endlichen Gruppen durch lineaire Substitusionen,Sitzungsber, Deutsch. Akad. Wiss. Berling(1897),171-172.

[8] M. Oudghiri, Additive mappings preserving the kernel or the range of operators, Extracta Math. 24, pp. 251-258, (2009).

[9] P. $\breve{S}$ emrl, Two characterizations of automorphisms on $B(X)$, Studia Math. 150, pp. 143-149, (1993).

\section{H. Benbouziane}

Departement of Industrial Engineering, National School of Applied Sciences, University Sidi Mohamed Ben Abdellah, 1796 Atlas Fes, Morocco

e-mail : benbhassan@yahoo.fr

\section{Y. Bouramdane}

Department of Mathematics, Faculty of Sciences DharMahraz Fes, University Sidi Mohamed Ben Abdellah, 1796 Atlas Fes, Morocco

e-mail : youssef.bouramdane1@usmba.ac.ma

and 
M. Ech-Chérif El Kettani

Department of Mathematics,

Faculty of Sciences DharMahraz Fes,

University Sidi Mohamed Ben Abdellah, 1796 Atlas Fes,

Morocco

e-mail : melkettani@yahoo.fr 